\title{
WIENER FUNCTIONALS ON SPACES OF LIE ALGEBRA VALUED 1-CURRENTS, AND UNITARY REPRESENTATIONS OF CURRENT GROUPS
}

\author{
JEAN MARION
}

\begin{abstract}
Well-known results about Brownian and Wiener functionals on abstract Wiener spaces are extended to Wiener functionals on the space of $\mathcal{G}$ valued 1-currents on a manifold $X$, where $\mathcal{G}$ is the Lie algebra of a compact semisimple Lie group $G$. We introduce a family of Shigekawa-Sobolev spaces of generalized Wiener functionals and on each of them one gets a regular representation of the current group $\mathcal{D}(X, G)$ of $G$-valued and compactly supported smooth mappings on $X$. Then, a kind of Weyl's construction is used to associate, to each Riemannian flag of $X$, a ring of non located and order I unitary representations of $\mathcal{D}(X, G)$ including the energy representations studied in [23], [14].
\end{abstract}

\section{Introduction}

If we should know a sufficiently large number of non trivial unitary representations of the current group $\mathcal{D}(X, G)$ it would be possible, as for finite dimensional Lie groups, to get a relevant non commutative harmonic analysis on it. Unfortunately, at the present time, our knowledge of the unitary dual of $\mathcal{D}(X, G)$ is rather poor, in spite of some efforts ([12], [6], [23], [14]).

In these works a class of non located and order 1 continuous unitary representations of $\mathcal{D}(X, G)$ are constructed, often called energy representations, irreducible when $\operatorname{dim}(X) \geq 3$; they are all realizable in a $L^{2}$-space of Wiener functionals on the space of $\mathcal{G}$-valued 1-currents on the manifold $X$, where $\mathcal{G}$ is the Lie algebra of the compact semisimple Lie group $G$.

The original motivation of the present work, consisting of to find a consistent enlargement of the class of unitary representations of $\mathcal{D}(X, G)$ actually known, leads then to begin by a detailed study of these Wiener functionals. The section $\mathrm{I}$ is devoted to the construction of $\mathcal{D}(X, G)$-invariant Euclidean structures on the space of $\mathcal{G}$-valued smooth 1 -forms with compact support on $X$ and Gaussian measures on the space $\mathcal{D}_{1}^{*}(X, \mathcal{G})$ of $\mathcal{G}$-valued 1-currents on $X$, associated with Riemannian flags. 
The section II is devoted mainly to the study of the spaces of $L^{2}$-Wiener functionals on $\mathcal{D}_{1}^{*}(X, \mathcal{G})$ in a way similar to the one used in [5] for the study of Brownian functionals associated to white noise, and the Wiener-Itô decomposition of such spaces is given. In section III we introduce a class of transforms, called $M$-transforms and which includes the Fourier-Wiener transform and the generalized Ornstein-Uhlenbeck semigroup; the study of its infinitesimal generator leads to the realization of a Hilbert-Sobolev space of Wiener functionals.

In section IV we extend this result by the introduction for each real number $\alpha>0$, of a semigroup of contractions, (the Ornstein-Uhlenbeck semigroup corresponding to the case $\alpha=1$ ) whose infinitesimal generator allows to construct a Shigekawa's model of Hilbert-Sobolev space of Wiener functionals, generalizing the situation described in [19], and denoted $L_{F, \alpha}^{2,1}$.

The section $\mathrm{V}$ is devoted to the construction and the study, for each $\alpha \geq 0$, of a regular (of course unitary) representation of $\mathcal{D}(X, G)$ on $L_{F, \alpha}^{2,1}$. A rather surprising fact, unknown in the case of finite dimensional Lie groups, is exhibited: on the subspace of polynomial Wiener functionals these various regular representation are equal and unitary with respect as well to the $L^{2}$ scalar product as to the various scalar products $L_{F, \alpha}^{2,1}$ of Sobolev type.

The section VI is devoted to the construction of non located unitary representations of order 1 of $\mathcal{D}(X, G)$; it appears that they are unitarily equivalent to the energy representations constructed in [14].

Lastly, in section VII, starting from the lot of representations constructed in section VI, and using a kind of generalization of the Weyl's construction, to each Riemannian flag of $X$ is associated a ring of non located unitary representations of order 1 of $\mathcal{D}(X, G)$ called generalized energy representations, including as very particular elements the energy representations given in [14].

\section{I. $\mathcal{G}$-valued 1 -currents and energy Gaussian measures}

In all this paper $X$ is a Riemannian manifold with finite dimension $d_{0}$, and $\mathcal{G}$ is the Lie algebra of a compact semisimple Lie group $G$, endowed with the Euclidean structure coming from the opposite of its Killing form. Ad will denote the adjoint representation of $G$ on $\mathcal{G}, N$ the set of non negative integers, and $\mathrm{N}^{*}$ the set of strictly positive integers.

1. The nuclear space of $\mathcal{G}$-valued 1 -forms and its energy scalar products.

a) Let $\mathcal{D}_{1}(X, \mathcal{G})$ be the space of all the $\mathcal{G}$-valued and compactly supported smooth I-forms on $X$; with its Schwartz topology it is a countably Hilbert nuclear space. The nuclear Lie group $\mathcal{D}(X, G)$ of all the $G$-valued and compactly supported smooth mappings on $X$ is a current group acting continuousiy on $\mathcal{D}_{1}(X, \mathcal{G})$ by a representation $V$ such that for all $g$ in $\mathcal{D}(X, G)$ and all $\omega$ in $\mathcal{D}_{1}(X, \mathcal{G}), V(g) \omega$ in the 1 -form:

$$
x \mapsto(V(g) \omega)(x)=\operatorname{Adg}(x) \cdot \omega(x), \quad x \in X .
$$


b) In order to endow $\mathcal{D}_{1}(X, \mathcal{G})$ with a positive definite inner product for which the operators $V(g)$ are unitary operators we shall need a geometrical object studied and used in [14], called a Riemannian flag (see also [13] for a summary of results).

Definition 1. A Riemannian flag of $X$ is a collection $F=\left(X_{k}, r_{k}\right)_{0 \leq k \leq p}$, with $0 \leq p \leq d_{0}$, such that:

- for each integer $k$ with $0 \leq k \leq p, X_{k}$ is a connected submanifold of $X$ with dimension $d_{0}-k$, and $r_{k}$ is a Riemannian metric of class $C^{1}$ on $X_{k}$;

$-X_{p} \subset \cdots \subset X_{k} \subset X_{k-1} \subset \cdots \subset X_{0}=X$.

Let $F=\left(X_{k}, r_{k}\right)_{0 \leq k \leq p}$ be a Riemannian flag of $X$; for each integer $k$ with $0 \leq k \leq p$ let us denote by $d x_{k}$ the volume measure on $X_{k}$, and $(,)_{k, x}$ the scalar product on the tangent space $T_{x} X_{k}$ of $X_{k}$ at $x$ with respect to the Riemannian metric $r_{k}$. One gets a scalar product $(,)_{k}$ on $\mathcal{D}_{1}\left(X_{k}, \mathcal{G}\right)$, defined for all $\lambda, \lambda^{\prime}$ in $\mathcal{D}_{1}\left(X_{k}, \mathcal{G}\right)$ by:

$$
\left(\lambda, \lambda^{\prime}\right)_{k}=\int_{X_{k}} \operatorname{tr}\left(\lambda^{\prime *}(x) \cdot \lambda(x)\right) d x_{k}
$$

where $t r$ is the trace operator, and where ${ }^{*}$ denotes here the adjoint operation according to the Euclidean structures on $T_{x} X_{k}$ and $\mathcal{G}$.

c) The energy scalar product $\left\langle,>_{F}\right.$ on $\mathcal{D}_{1}(X, \mathcal{G})$ associated to the Riemannian flag $F$ is then given by taking, for all $\omega, \omega^{\prime}$ in $\mathcal{D}_{1}(X, \mathcal{G})$ :

$$
\left\langle\omega, \omega^{\prime}>_{F}=\Sigma_{k=0}^{p}\left(\omega_{k}, \omega_{k}^{\prime}\right)_{k},\right.
$$

where $\omega_{k}$ and $\omega_{k}^{\prime}$ are the restrictions of $\omega$ and $\omega^{\prime}$ respectively to the submanifold $X_{k}, 0 \leq k \leq p$. We shall denote by $H^{F}$ the Hilbert completion of $\mathcal{D}_{1}(X, \mathcal{G})$ with respect to $\langle,\rangle_{F}$, and by $\left.\right|_{F}$ the corresponding norm.

Owing to the unitarity of the adjoint representation $A d$ of $G$ on $\mathcal{G}$, it follows that for all $g$ in $\mathcal{D}(X, G)$ the operators $V(g)$ extend into unitary operators on $H^{F}$, and then:

Lemma 1. For any Riemannian flag $F$ of $X, V$ is a continuous unitary representation of the current group $\mathcal{D}(X, G)$ on $H^{F}$.

Of course, $V$ is highly reducible, looking like a continuous sum of representations of $G$ on $\mathcal{G}$.

\section{The space of $\mathcal{G}$-valued 1-currents and its energy}

\section{Gaussian measures.}

a) Let $\mathcal{D}_{1}^{*}(X, \mathcal{G})$ be the dual space of $\mathcal{D}_{1}(X, \mathcal{G})$; it is the space of $\mathcal{G}$-valued 1-currents on $X$. In the present work it is the basic space on which the distribution of a generalized stochastic process will be located in order to expand a relevant analysis.

A well-known and standard result about dual spaces of countably Hilbert nuclear spaces allows to claim that for any Riemannian flag $F$ of $X$, the triple 
$\left[\mathcal{D}_{1}(X, \mathcal{G}), H^{F}, \mathcal{D}_{1}^{*}(X, \mathcal{G})\right]$ is a Gelf'and triple, i.e. an Hilbertian triad in the sense of [3]. We shall denote by $\left\langle,>\right.$ the pairing of $\mathcal{D}_{\mathrm{I}}(X, \mathcal{G})$ and its dual space.

The natural extension $V^{*}$ of the representation $V$ on $\mathcal{D}_{1}^{*}(X, \mathcal{G})$ is such that for all $g$ in $\mathcal{D}(X, G)$, all $\chi$ in $\mathcal{D}_{1}^{*}(X, \mathcal{G})$, and all $\omega$ in $\mathcal{D}_{1}(X, \mathcal{G})$ :

$$
\left\langle V^{*}(g) \chi, \omega\right\rangle=\left\langle\chi, V\left(g^{-1}\right) \omega\right\rangle \text {. }
$$

b) Notice that for each Riemannian flag $F$ of $X$ the mapping $C_{F}$ : $\mathcal{D}_{1}(X, \mathcal{G}) \rightarrow \mathbf{R}$ given by:

$$
C_{F}(\omega)=\exp \left[-\frac{1}{2}|\omega|_{F}^{2}\right], \quad \omega \in \mathcal{D}_{1}(X, \mathcal{G}),
$$

is a characteristic functional. From the Bochner-Minlos theorem it follows that there exists one and only one Gaussian measure $\mu_{F}$ on $\mathcal{D}_{1}^{*}(X, \mathcal{G})$ with mean zero, and Fourier transform $\hat{\mu}_{F}$ given by:

$$
\hat{\mu}_{F}(\omega)=\int_{\mathcal{D}_{i}^{*}(X, \mathcal{G})} \exp [i<\chi, \omega>] \mathrm{d} \mu_{F}(\chi)=C_{F}(\omega) .
$$

Moreover, $\mu_{F}$ satisfies the condition:

$$
\int_{\mathcal{D}_{i}^{*}(X, G)}\langle\chi, \omega\rangle \cdot\left\langle\chi, \omega^{t}\right\rangle d \mu_{F}(\chi)=\left\langle\omega, \omega^{\prime}\right\rangle_{F}
$$

for all $\omega, \omega^{\prime}$ in $\mathcal{D}_{1}(X, \mathcal{G})$.

$\mu_{F}$ will be called the energy Gaussian measure associated with the Riemannian flag $F$.

c) Notice that two Riemannian flags:

$F=\left(X_{k}, r_{k}\right)_{0 \leq k \leq p}$ and $F^{\prime}=\left(X_{k}^{\prime}, r_{k}^{\prime}\right)_{0 \leq k \leq p^{\prime}}$ are equal if and only if $p=p^{\prime}$, and for all $k=0,1, \ldots, p$, the Riemannian manifolds $\left(X_{k}, r_{k}\right)$ and $\left(X_{k}^{\prime}, r_{k}^{\prime}\right)$ are equal. Now let us recall the result of Feldman and Hajek asserting that two Gaussian measures are either equivalent or disjoint.

As a direct consequence of proposition II-2 given in [14] one gets:

Proposition 1. The energy Gaussian measures $\mu_{F}$ and $\mu_{F^{\prime}}$, associated with two different Riemannian fiags $F$ and $F^{\prime}$ are disjoint.

d) Now a crucial point is the behaviour of $\mu_{F}$ through the action of the current group $\mathcal{D}(X, G)$ by the representation $V^{*}$.

Proposition 2. Let $\mathcal{B}$ be the topological $\sigma$-field of $\mathcal{D}_{1}^{*}(X, \mathcal{G})$, and let $F$ be a Riemannian flag of $X$. For any element $g$ in $\mathcal{D}(X, G), V^{*}(g)$ is a $B$ measurable mapping from $\mathcal{D}_{1}^{*}(X, \mathcal{G})$ onto itself. Moreover $\mu_{F} \circ V^{*}(g)=\mu_{F}$.

Proof: The first part of the assertion follows from the fact that $V$ is a continuous unitary representation of $\mathcal{D}(X, G)$ on $H^{F}$. In order to prove the 
second part it suffices to compute the characteristic functional of the measure $\mu_{F} \circ V^{*}(g)$ and to see that it is equal to the characteristic functional $C_{F}$ of $\mu_{F}$ :

$$
\begin{aligned}
& \int_{\mathcal{D}_{1}^{*}(X, \mathcal{G})} \exp [i<\chi, \omega>] \cdot d\left(\mu_{F} \circ V^{*}(g)\right)(\chi)= \\
& =\int_{\mathcal{D}_{1}^{*}(X, \mathcal{G})} e^{i\langle\chi, \omega\rangle} \cdot d \mu_{F}\left(V^{*}(g) \chi\right)= \\
& =\int_{\mathcal{D}_{1}^{*}(X, \mathcal{G})} e^{i\left\langle V^{*}\left(g^{-1}\right) \chi, \omega\right\rangle} \cdot d \mu_{F}(\chi)=\int_{\mathcal{D}_{1}^{*}(X, g)} e^{i\langle\chi, V(g) \omega\rangle} d \mu_{F}(\chi)= \\
& =C_{F}(V(g) \omega)=\exp \left[-\frac{1}{2}|V(g) \omega|_{F}^{2}\right]=\exp \left[-\frac{1}{2} \mid \omega{ }_{F}^{2}\right]=C_{F}(\omega)
\end{aligned}
$$

by the unitary of the operators $V(g)$. Therefore $\mu_{F}$ is $\mathcal{D}(X, G)$-invariant.

\section{The space of $L^{2}-$ Wiener functionals on the $\mathcal{G}$-valued I-currents}

1. The spaces $L^{p}\left(\mathcal{D}_{1}^{*}(X, \mathcal{G}) ; h ; \mu_{F}\right)$.

Let $F$ be a Riemannian flag of $X$, let $\mathcal{B}_{F}$ be the completion of the topological $\sigma$-field $\mathcal{B}$ of $\mathcal{D}_{1}^{*}(X, \mathcal{G})$ (with respect to $\mu_{F}$ ), let $h$ be a separable Hilbert space with norm \|\|$_{h}$ and topological $\sigma$-field $\mathcal{B}_{h}$.

Definition 2. The measurable mappings from the measurable space $\left.\mathcal{D}_{1}^{*}(X, \mathcal{G}), \mathcal{B}_{F}\right)$ into the measurable space $\left(h, \mathcal{B}_{h}\right)$ are called $h$-valued Wiener functionals on $\mathcal{D}_{1}^{*}(X, \mathcal{G})$.

The above definition generalizes in a natural way the concept of Brownian functional studied in [5], [10], [17].

Of course, two $h$-valued Wiener functionals are considered in the same class whenever they are equal $\mu_{F}$-almost everywhere; so there is no difficulty to define for all real $p \geq 1$ the space $L^{p}\left(\mathcal{D}_{1}^{*}(X, \mathcal{G}) ; h ; \mu_{F}\right)$ of $h$-valued Wiener functionals $\Phi$ such that the mapping $\chi \mapsto\|\Phi(\chi)\|_{h}^{p}$ is $\mu_{F}$-integrable, the corresponding norm being given by

$$
\|\Phi\|_{L^{p}}=\left(\int_{\mathcal{D}_{1}^{*}(X, G)}\|\Phi(\chi)\|_{h}^{p} \cdot d \mu_{F}(\chi)\right)^{1 / p} .
$$

In this section we are mainly interested by the Hilbert spaces $L^{2}\left(\mathcal{D}_{1}^{*}(X, \mathcal{G}) ; h\right.$; $\left.\mu_{F}\right)$ and more particularly by $L^{2}\left(\mathcal{D}_{1}^{*}(X, \mathcal{G}) ; \mathrm{C} ; \mu_{F}\right)$ which will be more simply denoted $L_{F}^{2}$; its elements will be called $L^{2}$-Wiener functionals.

\section{Polynomial Wiener functionals.}

Formula (7) shows that for any 1 -form $\omega$ in $\mathcal{D}_{1}(X, \mathcal{G})$ the mapping $\chi \mapsto\langle\chi, \omega\rangle$ is a Gaussian random variable with mean zero and variance $|\omega|_{F}^{2}$; therefore, for any positive integer $n$ one has

$$
\int_{\mathcal{D}_{i}^{*}(X, \mathcal{G})}|<\chi, \omega>|^{2 n} \cdot \mu_{F}(\chi)=(2 n) !(n !)^{-1} \cdot 2^{-n} \cdot|\omega|_{F}^{2 n}
$$


it follows that the mapping $\chi \mapsto\langle\chi, \omega\rangle^{n}$ belongs to $L_{F}^{2}$ for all positive integer $n$, and all element $\omega$ in $\mathcal{D}_{1}(X, \mathcal{G})$.

Now, using the Schwarz inequality and a standard argument, one easily proves that for any collection $\left(\omega_{1}, \ldots, \omega_{q}\right)$ of elements in $\mathcal{D}_{1}(X, \mathcal{G})$ and any collection $\left(n_{1}, \ldots, n_{q}\right)$ of non negative integers the mapping $\chi \mapsto<\chi, \omega_{1}>^{n_{1}} \ldots$ $\left\langle\chi, \omega_{q}\right\rangle^{n_{q}}$ belongs to $L_{F}^{2}$.

Definition 3. A $C$-valued Wiener functional $\Phi$ is called a polynomial Wiener functional if there exist a positive integer $q, q$ elements $\omega_{1}, \ldots, \omega_{q}$ in $\mathcal{D}_{1}(X, \mathcal{G})$, and a polynomial function $p: \mathbf{R}^{q} \rightarrow C$ such that, for all $\chi$ in $\mathcal{D}_{1}^{*}(X, \mathcal{G})$ :

$$
\Phi(\chi)=p\left(<\chi, \omega_{1}>, \ldots,<\chi, \omega_{q}>\right) .
$$

We notice, from the above discussion, that the space $P$ of all the $C$-valued polynomial Wiener functionals is an algebra contained in $L_{F}^{2}$.

Remark. Let $h$ be a separable Hilbert space; the elements of $P \otimes h$ are the $h$-valued polynomial Wiener functionals on $\mathcal{D}_{1}^{*}(X, \mathcal{G})$.

A Riemannian flag $F$ of $X$ being given, let us consider a complete orthonormal system $\left(\omega_{i}\right)_{i \in N^{*}}$ of $H^{F}$ with all the $\omega_{i}$ in $\mathcal{D}_{1}(X, \mathcal{G})$. Owing to the above discussion and from the density of $\mathcal{D}_{1}(X, \mathcal{G})$ in $\left(H^{F}\right)^{*}=H^{F}$ it follows easily that:

Proposition 3. For all positive integer $p, P \otimes h$ is a dense linear subspace of $L^{P}\left(\mathcal{D}_{1}^{*}(X, \mathcal{G}) ; h_{j}, \mu_{F}\right)$. In particular the algebra $P$ is dense in $L_{F}^{2}$.

\section{Exponential Wiener functionals.}

a) Definition 4: A $C$-valued Wiener functional $\Phi$ is said an exponential Wiener functional if there exist a complex number $\alpha$ and a 1 -form $\omega$ in $\mathcal{D}_{1}(X, \mathcal{G})$ such that $\Phi(\chi)=\exp [\alpha<\chi, \omega\rangle]$ for all $\chi$ in $\mathcal{D}_{1}^{*}(X, \mathcal{G})$.

Lemma 2. All the exponential Wiener functionals are $L^{2}-$ Wiener functionals.

Proof: The assertion follows from the fact that: $\exp [\alpha<\chi, \omega>]=$ $\sum_{n=0}^{\infty} \frac{\alpha^{n}}{n !}\langle\chi, \omega\rangle^{n}$, and that the mappings $\chi \rightarrow\langle\chi, \omega\rangle^{n}$ belong to $L_{F}^{2}$.

b) To each $\omega \operatorname{in} \mathcal{D}_{1}(X, \mathcal{G})$ let us associate the exponential Wiener functional $\hat{\omega}: \chi \mapsto \exp [i<\chi, \omega\rangle]$. We notice that $\mu_{F}(\hat{\omega})=\hat{\mu}_{F}(\omega)=C_{F}(\omega)$, and that the complex vector space $E$ spanned by all the $\hat{\omega}$ is an algebra. More precisely:

Proposition 4. The algebra $E$ is dense in $L_{F}^{2}$.

Proof: It is similar to the one given for exponential Brownian functionals given in [5], theorem 4.1. By lemma 2 we have $E \subset L_{F}^{2}$. Now let us recall that $\mathcal{B}$ denotes the topological $\sigma$-field of $\mathcal{D}_{1}^{*}(X, \mathcal{G})$ and that we have selected a complete orthonormal system $\left(\omega_{k}\right)_{k \in N^{*}}$ in $H^{F}$, the $\omega_{k}$ being in $\mathcal{D}_{1}(X, \mathcal{G})$. 
For each positive integer $n$, let us consider the sub- $\sigma$-field of $B$ generated by the functionals $\chi \mapsto<\chi, \omega_{k}>, 1 \leq k \leq n$, and denoted $\mathcal{B}_{n} .\left(\mathcal{B}_{n}\right)_{n \in N^{-}}$is an increasing sequence converging to $\mathcal{B}$.

In order to prove the assertion it suffices to show that any element $\Phi$ in $L_{F}^{2}$ which is orthogonal to all the elements of the form $\chi \mapsto \Pi_{k=1}^{n} \exp \left[\mathrm{it}_{k}\right.$ $\left\langle\chi, \omega_{k}\right\rangle$ ] for all positive integer $n$, and all $t_{1}, \ldots, t_{n}$ in $\mathbf{R}$, is necessarily zero $\mu_{F}$-almost everywhere.

So, let us assume that for any positive integer $n$ and any sequence $\left(t_{1}, \ldots, t_{n}\right)$ of real numbers the Wiener functional $\Phi$ satisfies:

$$
\hat{\Phi}_{n}=\int_{\mathcal{D}_{1}^{*}(X, G)} \Pi_{k=1}^{n} \exp \left[i t_{k}<\chi, \omega_{k}>\right] \cdot \bar{\Phi}(\chi) d \mu_{F}(\chi)=0,
$$

and let us denote by $E\left(\bar{\Phi} \mid B_{n}\right)$ the conditional expectation of $\bar{\Phi}$ with respect to the sub $-\sigma$-field $\mathcal{B}_{n}$. One has:

$$
\hat{\Phi}_{n}=\int_{\mathcal{D}_{i}^{*}(X, \mathcal{G})} \exp \left[i<\chi, \Sigma_{k=1}^{n} t_{k} \omega_{k}>\right] \cdot E\left(\bar{\Phi} \mid \mathcal{B}_{n}\right) d \mu_{F}(\chi)=0 .
$$

The Fourier transform of $\chi \mapsto \mathrm{E}\left(\bar{\Phi}(\chi) \mid \mathcal{B}_{n}\right)$ is then zero on the subspace spanned by $\left(\omega_{1}, \ldots, \omega_{n}\right)$; therefore, for all positive integer $n, E\left(\bar{\Phi} \mid \mathcal{B}_{n}\right)$ is zero $\mu_{F}$-a.e. $\left(\mathcal{B}_{n}\right)_{n}$ being increasing sequence converging to the $\sigma$-field $\mathcal{B}$, it follows that $\mathrm{E}(\bar{\Phi} \mid \mathcal{B})=\bar{\Phi}=0, \mu_{F}-$ a.e. The assertion is then proved.

4. The Wiener-Itô decomposition of $L_{F}^{2}$.

a) Let us recall first a well-known result: the system of mappings $\left(\sqrt{n !} \cdot H_{n}\right)_{n \in N}$, where $H_{n}$ denotes the Hermite polynomial function given by:

$$
H_{n}(t)=(-1)^{n}(n !)^{-1} \exp \left(t^{2} / 2\right) \frac{d^{n}}{d t^{n}}\left[\exp \left(-t^{2} / 2\right)\right], t \in \mathbf{R},
$$

is a complete orthonormal system in $L^{2}\left(\mathbf{R} ;(2 \pi)^{-1 / 2} \exp \left(-t^{2} / 2\right) d t\right.$ ) (see e.g. [16]).

Let $\wedge$ be the set of sequences $\nu=\left(\nu_{k}\right)_{k \in N^{*}}$ of elements of $N$ such that $\nu_{k}=0$ except for finitely many $k$ 's. For such an element $\nu=\left(\nu_{k}\right)_{k \in N^{*}}$ in $\wedge$ we will denote:

$$
|\nu|=\sum_{n=1}^{\infty} \nu_{k} ; \quad \nu !=\Pi_{k=1}^{\infty} \nu_{k} !
$$

For all $n$ in $N, \wedge_{n}$ will denote the set $\{\nu \in \wedge /|\nu|=n\}$.

Now a Riemannian fiag $F$ of $X$ being given, we select a complete orthonormal system $\left(\omega_{k}\right)_{k \in N^{*}}$ of $H^{F}$, with all the $\omega_{k}$ in $\mathcal{D}_{1}(X, \mathcal{G})$.

Definition 5: For all $\nu$ in $\wedge$, the mapping $h_{\nu}$ defined on $\mathcal{D}_{1}^{*}(X, \mathcal{G})$ by:

$$
h_{\nu}(\chi)=\prod_{k} H_{\nu_{k}}\left(<\chi, \omega_{k}>\right)
$$

is called a Fourier-Hermite polynomial Wiener functional.

We have to give now the Wiener chaos decomposition of $L_{F}^{2}$; such a decomposition is well-known in the case of the space of $L^{2}$-Brownian functionals ([5]) and in the case of $L^{2}$-functionals on an abstract Wiener space ([19]). 
Proposition 5. The collection $\left(\sqrt{\nu !} h_{\nu}\right)_{\nu \in \Lambda}$ is a complete orthonormal system in $L_{F}^{2}$. For each $n$ in $\mathrm{N}$ let us denote by $Z_{n}^{F}$ the closed subspace of $L_{F}^{2}$ spanned by the family $\left(\sqrt{\nu !} h_{\nu}\right)_{\nu \in \wedge_{n}}$; one has the orthogonal direct sum, called the Wiener-Itô decomposition:

$$
L_{F}^{2}=\Sigma_{n=0}^{\infty} \oplus X_{n}^{F} .
$$

The proof is standard and we omit it. Of course the Wiener-Itô decomposition of $L_{F}^{2}$ is independent of a particular choice of the orthogonal system $\left(\omega_{k}\right)_{k \in N^{*}}$ of $H^{F}$.

Remark: Let us consider the symmetric Fock space $S\left(H^{F}\right)$ constructed with $H^{F}$ as one particle space:

$$
S\left(H^{F}\right)=\Sigma_{n=0}^{\infty} \oplus\left(H^{F}\right)^{\otimes n, s}
$$

where the sum is taken with orthogonal components, and where $\left(H^{F}\right)^{\otimes n, s}$ denotes the space of symmetric $n$-tensors on $H^{F}$. As it is well-known there is a natural Hilbert isomorphism from $S\left(H^{F}\right)$ onto $L_{F}^{2}$ ([4]). It is easy to see that for each integer $n$, this isomorphism restricts to a Hilbert isomorphism from $\left(H^{F}\right)^{\otimes n, s}$ onto $Z_{n}^{F}$.

We shall denote by $p_{n}^{F}$ the orthogonal projection from $L_{F}^{2}$ onto $Z_{n}^{F}, n \in \mathrm{N}$.

\section{III. $M$-transforms; Fourier-Wiener transform; generalized Ornstein-Uhlenbeck semigroups}

\section{1. $M$-transforms.}

For a complex number $z \neq 0$ we shall denote by $\sqrt{z}$ the complex number such that $(\sqrt{z})^{2}=z$ and such that arg $(\sqrt{z})$ is in $[0, \pi]$. For each non zero complex number $z$ we define the transform $M_{z}^{F}$ from $L^{\mathbb{I}}\left(\mathcal{D}_{1}^{*}(X, \mathcal{G}) ; \mathrm{C} ; \mu_{F}\right)$ into itself by:

$$
M_{z}^{F} \Phi(\chi)=\int_{\mathcal{D}_{i}^{*}(X, \xi)} \Phi\left(z \chi+\sqrt{1-z^{2}} \theta\right) d \mu_{F}(\theta),
$$

where $F$ is a given Riemannian flag of $X$. The $M_{z}^{F}, z \in \mathrm{C}-\{0\}$, will be called $M$-transforms. Particular cases of such transforms have been considered: the Fourier-Wiener transform introduced by Cameron and Martin ([2]) on the space $S^{\prime}$ of tempered distributions, and, more recently, the Ornstein-Uhlenbeck operator on an abstract Wiener space $(\mathbf{1 5}])$.

The basic properties of the so-called $M$-transforms come from the wellknown property of Hermite polynomials (see e.g. [16], chap. IV), namely:

$$
\begin{aligned}
& \frac{1}{\sqrt{2 \pi}} \int_{\mathbf{R}} H_{n}\left(z x / \sqrt{1-z^{2}} y\right) \exp \left(-y^{2} / 2\right) d y=z^{n} H_{n}(x), \\
& z \in \mathbb{C}-\{0\}, n \in N .
\end{aligned}
$$

From the definition of Fourier-Hermite polynomials and from the above formula it follows that: 
Lemma 3. Let $F$ be a Riemannian flag of $X$, and let $z$ be any non zero complex number. For all $\nu$ in $\wedge_{n}, n \in \mathrm{N}$ one has:

$$
M_{z}^{F} h_{\nu}=z^{n} h_{\nu} \text {. }
$$

Notice that $M_{z}^{F}$ is a linear operator on $L_{F}^{2}$. As a direct consequence of lemma $S$ one gets:

Lemma 4. Let $F$ and $z$ be as in lemma 9. One has:

$$
M_{z}^{F}=\sum_{n=0}^{\infty} z^{n} p_{n}^{F} .
$$

In particular, for each integer $n$ we have the commutation:

$$
M_{z}^{F} \cdot p_{n}^{F}=p_{n}^{F} \cdot M_{z}^{F}
$$

\section{The Fourier-Wiener transform.}

Definition 6. Let $F$ be a Riemannian flag of $X$; the $M$-transform $M_{i}^{F}$ is called the Fourier-Wiener transform associated to $F$, and denoted $\mathcal{J}^{F}$.

As a direct consequence of lemma 4 we have:

Proposition 6. $\mathcal{J}^{F}$ is a unitary operator on $L_{F}^{2}$ satisfying the equality: $\mathcal{J}^{F}=\sum_{n=0}^{\infty} i^{n} p_{n}^{F}$. In particular $\left(\mathcal{J}^{F}\right)^{4}=I\left(I=\right.$ identity operator on $\left.L_{F}^{2}\right)$. Notice that, by definition of the transformations $M_{z}^{F}$, one has, for all $\Phi$ in $L_{F}^{2}$ :

$$
\mathcal{J}^{F} \Phi(\chi)=\int_{\mathcal{D}_{1}^{*}(X, \mathcal{G})} \Phi(i \chi+\sqrt{2} \theta) d \mu_{F}(\theta)
$$

Let us compute now the Fourier-Wiener transform of the basic Wiener functionals; we recall that for $\omega$ in $\mathcal{D}_{1}(X, \mathcal{G}), \hat{\omega}$ denotes the exponential Wiener functional $\chi \mapsto \exp [i<\chi, \omega>]$.

Proposition 7. Let $n$ be in $N$, let $\omega$ be in $\mathcal{D}_{1}(X, \mathcal{G})$ and let $\nu$ be in $\wedge_{n}$. Then:

(i) $\mathcal{J}^{F} h_{\nu}=i^{n} h_{\nu}$;

(ii) $\mathcal{J}^{F} \hat{\omega}=\exp \left(-|\omega|_{F}^{2}\right) \exp [-<\cdot, \omega>]$.

Proof: (i) is a trivial consequence of proposition 5 and lemma 3.

(ii) For all $\chi$ in $\mathcal{D}_{1}^{*}(X, \mathcal{G})$ one has:

$$
\begin{aligned}
& \mathcal{J}^{F} \hat{\omega}(\chi)=\int_{\mathcal{D}_{i}^{*}(X, G)} \exp [i<i \chi+\sqrt{2} \theta, \omega>] d \mu_{F}(\theta)= \\
& \exp \left[-\langle\chi, \omega>] \int_{\mathcal{D}_{1}^{*}(X, G)} \exp [i<\theta, \sqrt{2} \omega>] d \mu_{F}(\theta)=\right. \\
& \exp [-\langle\chi, \omega\rangle] C_{F}(\sqrt{2} \omega)=\exp \left[-|\omega|_{F}^{2}\right] \exp [-<\chi, \omega>]
\end{aligned}
$$

An immediate consequence of lemma 4 , proposition 7 (i), and the definitions is: 
Corollary, (i) For all $n$ in $\mathrm{N}, p_{n}^{F} \cdot \mathcal{J}^{F}=\mathcal{J}^{F} \cdot p_{n}^{F}$;

(ii) for any non zero complex number $z$ one has:

$$
M_{z}^{F} \cdot \mathcal{J}^{F}=\mathcal{J}^{F} \cdot M_{z}^{F}
$$

\section{F-Ornstein-Uhlenbeck semigroup and operator.}

Let $F$ be a Riemannian flag of $X$, and let us consider the family $\left(F_{t}\right)_{t \geq 0}$ of $M$-transforms defined for all real numbers $t \geq 0$ by:

$$
F_{t}=M_{e^{-t}}^{F}
$$

For all $\Phi$ in $L_{F}^{2}$ we have then:

$$
F_{t} \Phi(\chi)=\int_{D_{i}^{;}(X, \mathcal{G})} \Phi\left(e^{-t} \chi+\sqrt{1-e^{-2 t}} \theta\right) d \mu_{F}(\theta) .
$$

Notice that from lemma 3 , for all integers $n \geq 0$ and all $\nu$ in $\wedge_{n}$ one has:

$$
F_{t} h_{\nu}=e^{-n t} h_{\nu}, \quad t \geq 0
$$

Notice also that for all $s, t$ positive real numbers $F_{s} \cdot F_{t}=F_{s+t}$ and that $0 \leq$ $e^{-n t} \leq 1$. From lemma 4 , corollary of proposition 7 and the above discussion if follows that:

\section{Proposition 8.}

(i) $\left(F_{t}\right)_{t \geq 0}$ is a contraction semigroup of $M$-transforms on $L_{F}^{2}$.

(ii) $F_{t}=\sum_{n=0}^{\infty} e^{-n t} p_{n}^{F}$, and for all integers $n \geq 0$ :

$$
Z_{n}^{F}=p_{n}^{F}\left(L_{F}^{2}\right)=\left\{\Phi \in L_{F}^{2} / F_{t} \Phi=e^{-n t} \Phi \text { for all } t>0\right\}
$$

(iii) $F_{i} \cdot \mathcal{J}^{F}=\mathcal{J}^{F} \cdot F_{i}$.

Definition 7. $\left(F_{t}\right)_{t \geq 0}$ will be called the Ornstein-Uhlenbeck semigroup on $L_{F}^{2}$, or $F$-Ornstein-Uhlenbeck semigroup.

Notice that usual Ornstein-Uhlenbeck semigroups in infinite dimension axe defined on $L^{p}$-spaces based on abstract Wiener spaces (see e.g. [19]).

The computation of the infinitesimal generator $L^{F}$ of $\left(F_{t}\right)_{t \geq 0}$, called the $F$-Ornstcin-Uhlenbeck operator is very easy:

$$
L^{F}=\frac{d}{d t}\left(F_{t}\right)_{t=0}=\frac{d}{d t}\left(\Sigma_{n=0}^{\infty} e^{-n t} p_{n}^{F}\right)_{t=0}=\Sigma_{n=1}^{\infty}(-n) p_{n}^{F}
$$

It follows that: 
Proposition 9. The infinitesimal generator $L^{F}$ of $\left(F_{t}\right)_{t \geq 0}$ is given by $L^{F}=$ $\Sigma_{n=1}^{\infty}(-n) p_{n}^{F}$, with domain $D\left(L^{F}\right)=\left\{\Phi \in L_{F}^{2} / \Sigma_{n=1}^{\infty} n^{2}\left\|p_{n}^{F} \Phi\right\|_{L^{2}}^{2}<+\infty\right\}$.

Remark. The space $P$ of polynomial Wiener functionals is contained in $D\left(L^{F}\right)$ because for a Fourier-Hermite polynomial Wiener functional $h_{\nu}, \nu \in \Lambda$, $p_{n}^{F} h_{\nu}$ is zero if $|\nu| \neq n$ and is equal to $h_{\nu}$ if $|\nu|=n$; as ||$\sqrt{\nu !} h_{\nu} \|_{L^{2}}=1$ it follows that:

$$
\sum_{n=1}^{\infty} n^{2}\left\|p_{n}^{F} h_{\nu}\right\|_{L^{2}}^{2}=|\nu|^{2}(\nu !)^{-1}<+\infty .
$$

Therefore, using standard arguments, from the above remark and proposition 9 it follows that:

Proposition 10. $L^{F}$ extends to a Hermition operator with domain containing the algebraic sum $\sum_{n=0}^{\infty} Z_{n}^{F}$, and for each integer $n \geq 0, Z_{n}^{F}$ is in the eigenspace corresponding to the eigenvalue $-n$.

Proposition 11. $L^{F}$ extends to the exponential Wiener functionals; moreover, for all $z$ in $\mathcal{C}$ and all $\omega$ in $\mathcal{D}_{1}(X, \mathcal{G})$ one has:

$$
\left.L^{F} \exp [z<\chi, \omega>]=\left[z^{2}|\omega|_{F}^{2}-z<\chi, \omega>\right] \exp [z<\chi, \omega\rangle\right]
$$

for all $\chi$ in $\mathcal{D}_{1}^{*}(X, \mathcal{G})$.

Proof: For any polynomial Wiener functional $\Phi$ its Fréchet derivative $D \Phi(\chi)$ at the 1 -current $\chi$ is the element of $\mathcal{D}_{1}(X, \mathcal{G})$ defined by:

$$
<\theta, D \Phi(\chi)>=\frac{d}{d t}(\Phi(\chi+t \theta))_{t=0}, \quad \theta \in \mathcal{D}_{1}^{*}(X, \mathcal{G})
$$

Notice that $D \Phi: \chi \mapsto D \Phi(\chi)$ belongs to $P \otimes H^{F}$; in the same way we define $D^{2} \Phi$ as $D(D \Phi)$, and therefore as an element in $P \otimes H^{F} \otimes H^{F}$. A complete orthonormal system $\left(\omega_{k}\right)_{k \in \mathrm{N}^{*}}$ in $H^{\bar{F}}$ being given, the trace of $D^{2} \Phi(\chi), \chi$ in $\mathcal{D}_{1}^{*}(X, \mathcal{G})$ is then given by the standard formula:

$$
\text { trace }\left[D^{2} \Phi(\chi)\right]=\sum_{n=1}^{\infty}\left\langle D^{2} \Phi(\chi), \omega_{n} \otimes \omega_{n}\right\rangle
$$

that we can rewrite:

$$
\text { trace }\left[D^{2} \Phi(\chi)\right]=\int_{\mathcal{D}_{i}^{*}(X, \mathcal{G})}<D^{2} \Phi(\chi), \theta \otimes \theta>d \mu_{F}(\theta) .
$$

Now, using a similar argument of one that is used in the case of usual OrnsteinUhlenbeck operator, one gets, for all $\Phi$ in $P$ :

$$
L^{F} \Phi(\chi)=\operatorname{trace}\left[D^{2} \Phi(\chi)\right]-<\chi, D \Phi(\chi)>.
$$


Let us consider now the exponential Wiener functional $\hat{\omega}_{z}: \chi \mapsto \exp [z<$ $\chi, \omega>\}, z$ and $\omega$ being given in $C$ and $\mathcal{D}_{1}(X, \mathcal{G})$ respectively. One has:

$\hat{\omega}_{z}=\Sigma_{n=0}^{\infty} \frac{z^{n}}{n !}\langle\cdot, \omega\rangle^{n}$. Therefore we can extend $L^{F}$ to $\hat{\omega}_{z}$ by taking:

$\left.L^{F} \hat{\omega}_{z}=\Sigma_{n=0}^{\infty} \frac{x^{n}}{n !} L^{F}(<\cdot, \omega\rangle^{n}\right)$; of course, (22) remains valid for $\Phi=\hat{\omega}_{z}$ and we can use this formula to compute $L^{F} \hat{\omega}_{z}$. For all $\theta$ in $\mathcal{D}_{1}^{*}(X, \mathcal{G})$ and all $\chi$ in $\mathcal{D}_{1}^{*}(X, \mathcal{G})$ :

$$
\begin{aligned}
\left\langle\theta, D \hat{\omega}_{z}(\chi)>\right. & =\frac{d}{d t}(\exp [z<\chi+t \theta, \omega>])_{t=0} \\
& =\exp [z<\chi, \omega>] \frac{d}{d t}\left(e^{t z<\theta, \omega>}\right)_{t=0} \\
& =z<\theta, \omega>\hat{\omega}_{z}(\chi)
\end{aligned}
$$

In particular $\left\langle\chi, D \hat{\omega}_{z}(\chi)\right\rangle=z\langle\chi, \omega\rangle \cdot \hat{\omega}_{z}(\chi) \cdot\left({ }^{* *}\right)$

In the same way, taking $\theta, \theta^{\prime}$ in $\mathcal{D}_{1}^{*}(X, \mathcal{G})$, and using $\left(^{*}\right)$ for the computation of $D^{2} \hat{\omega}_{z}(\chi)$ on gets:

$$
\left\langle D^{2} \hat{\omega}_{z}(\chi), \theta \otimes \theta^{\prime}\right\rangle=z^{2}\langle\theta, \omega\rangle \cdot\left\langle\theta^{\prime}, \omega\right\rangle \cdot \hat{\omega}_{z}(\chi) .
$$

Therefore trace $\left[D^{2} \hat{\omega}_{z}(\chi)\right]=z^{2} \cdot \hat{\omega}_{z}(\chi) \int_{\mathcal{D}_{i}^{*}(X, \mathcal{G})}\langle\theta, \omega\rangle \cdot\langle\theta, \omega\rangle d \mu_{F}(\theta)=$ $z^{2} \hat{\omega}_{z}(\chi)|\omega|_{F}^{2}$ by formula $(7)$, I. Now taking into account this formula with $\left(^{*}\right)$, the formula (22) gives:

$$
L^{F} \hat{\omega}_{z}(\chi)=\left(z^{2}|\omega|_{F}^{2}-z<\chi, \omega>\right) \hat{\omega}_{z}(\chi)
$$

\section{Shigekawa-Hilbert-Sobolev spaces of generalized Wiener functionals on $\mathcal{D}_{1}^{*}(X, \mathcal{G})$}

In the last part of the above section the formula (22) shows that $L^{F}$ looks like a Laplace-Beltrami operator. In particular proposition 11 is a generalization of proposition 5.13 in [5] giving a similar result for the so-called Laplacian introduced by Umemura ([21]) which corresponds to the infinitesimal generator of the usual Ornstein-Uhlenbeck semigroup.

\section{The Hilbert-Sobolev space $L_{F}^{2,1}$.}

Let $F$ be a Riemannian flag of $X$; the Ornstein-Uhlenbeck operator $L^{F}=$ $-\sum_{n=0}^{\infty} n p_{n}^{F}$ is a negative definite self-adjoint operator on $L_{F}^{2}$ with domain containing the dense subspace $P$ of $L_{F}^{2}$ consisting of the polynomial Wiener functionals.

Let us consider the operator $C^{F, 1}$ defined on $P$ by:

$$
C^{F, 1}=\sqrt{-L^{F}}=\Sigma_{n=0}^{\infty} \sqrt{n} p_{n}^{F} .
$$


The operator $C^{F, 1}$ looks like the Cauchy operator defined as the square root of the opposite of the ustral Laplace-Beltrami operator. It allows to define a new scalar product $<,>_{L^{2,1}}$ on $P$ by:

$$
<\Phi, \psi>_{L^{2,1}}=<\Phi, \psi>_{L^{2}}+<C^{F, 1} \Phi, C^{F, 1} \psi>_{L^{2}} .
$$

Of course, $\langle,\rangle_{L^{2}}$ and $\left.<,\right\rangle_{L^{2,2}}$ are not equivalent because $C^{F, \pm}$ is unbounded, although they are equivalent on each subspace $p \cap Z_{n}^{F}$.

Formally, the scalar product given by (24) looks like the classical Sobolev scalar product of $L^{2,1}$ type. As it is well-known, in the case of Wiener functionals on an abstract Wiener space of infinite dimension there are two ways in order to define frames of Sobolev type. The one uses Fréchet derivatives and leads to the Shigekawa's model of Sobolev space; the other, using stochastic Gateaux derivatives, leads to the Kusuoka-Stroock's model of Sobolev space ([18], [11]). In fact it is proved in [20], theorem 3.1, that these two ways lead to the same Sobolev spaces. In the generalization that we shall give in the next section of such spaces we cannot claim that the two ways lead to the same spaces.

We shall denote by $L_{F}^{2,1}$ the Hilbert completion of $P$ with respect to the scalar product $<,>_{L^{2,2}}$.

\section{The Shigekawa-Hilbert-Sobolev spaces $L_{F, \alpha}^{2, \frac{1}{2}}$.}

We generalize here the situation described in 1). We select a Riemannian flag $F$ of $X$. Each element $\alpha$ in the set $\mathrm{R}^{+*}$ of strictly positive real numbers gives rise to a contraction semigroup $\left(F_{t}^{\alpha}\right)_{t \geq 0}$ on $L_{F}^{2}$, with $F_{t}^{\alpha}$ given by:

$$
F_{t}^{\alpha}=\Sigma_{n=0}^{\infty} e^{-n^{\alpha}} t_{n}^{F} \text {. }
$$

Notice that the $F$-Ornstein-Uhlenbeck semigroup given in definition 7 corresponds to the case $\alpha=1$.

The corresponding infinitesimal generator $F^{F, \alpha}$ is given by:

$$
L^{F, \alpha}=\frac{d}{d t}\left(F_{t}^{\alpha}\right)_{t=0}=\Sigma_{n=0}^{\infty}\left(-n^{\alpha}\right) p_{n}^{F},
$$

with domain $D\left(L^{F, \alpha}\right)$ :

$$
D\left(L^{F, \alpha}\right)=\left\{\Phi \in L_{F}^{2} / \Sigma_{n=1}^{\infty} n^{2 \alpha}\left\|p_{n}^{F} \Phi\right\|_{L^{2}}^{2}<\infty\right\} .
$$

Notice that the operators $L^{F, \alpha}$ are unbounded, negative definite, and selfadjoint on $L_{F}^{2}$. Moreover:

Lemma 5. For any $\alpha$ in $\mathrm{R}^{+*}, D\left(L^{F, \alpha}\right)$ contains the space $P$ of polynomial Wiener functionals and also the exponential Wiener functionals.

Proof: Let $\nu$ be in $\Lambda$, and let $n$ be in $\mathrm{N}$; then $p_{n}^{F}\left(h_{\nu}\right)=0$ if $|\nu| \neq n$, and $p_{n}^{F}\left(h_{\nu}\right)=h_{\nu}$ if $|\nu|=n$. It follows that $\sum_{n=1}^{\infty} n^{2 \alpha}\left\|p_{n}^{F}\left(h_{\nu}\right)\right\|_{L^{2}}^{2}=|\nu|^{2 \alpha}(\nu !)^{-1}$ by proposition 5 . Therefore $h_{v}$ belongs to $D\left(L^{F, \alpha}\right)$ and then $p \subset D\left(L^{F, \alpha}\right)$. 
Now the same argument that the one used in proposition 11 allows to claim that $L^{F, \alpha}$ extends to exponential Wiener functionals.

Let us consider now the square root $C^{F, \alpha}$ of the positive definite selfadjoint operator $-L^{F, \alpha}$ :

$$
C^{F, \alpha}=\Sigma_{n=1}^{\infty} n^{\alpha / 2} p_{n}^{F}=\sqrt{-L^{F, \alpha}}
$$

This generalized Cauchy operator allows to get a new scalar product $<,>_{L_{\alpha}^{2,1}}$ on $P$ by:

$$
\begin{aligned}
& <\Phi, \psi>_{L_{\alpha}^{2,1}}=\left\langle\Phi, \psi>_{L^{2}}+\left\langle C^{F, \alpha} \Phi, C^{F, \alpha} \psi\right\rangle_{L^{2}},\right. \\
& \text { with } \Phi, \psi \text { in } P .
\end{aligned}
$$

Moreover:

Lemma 6. Let $\alpha, \alpha^{\prime}$ in $\mathbf{R}^{+*}$ with $\alpha \neq \alpha^{\prime}$; then $\left\langle,>_{L_{\alpha^{\prime}}^{2,1}}\right.$ and $<,>_{L_{\alpha^{\prime}}^{2,1}}$ are non equivalent on $P$.

Proof: The assertion follows from the fact that, for a given $\nu$ in $\wedge$, if we suppose that $\alpha^{\prime}=\alpha+\beta$ with $\beta>0$, then, with $n=|\nu|$,

$$
\begin{aligned}
& <C^{F, \alpha+\beta} \sqrt{\nu !} h_{\nu}, C^{F, \alpha+\beta} \sqrt{\nu !} h_{\nu}>_{L^{2}}= \\
& n^{\alpha+\beta}=n^{\beta}<C^{F, \alpha} \sqrt{\nu !} h_{\nu}, C^{F, \alpha} \sqrt{\nu !} h_{\nu}>_{L^{2}} .
\end{aligned}
$$

Deffnition 8 . The Hilbert completion of $P$ with respect to $\langle,\rangle_{L_{\alpha}^{2.1}}$, denoted $L_{F, \alpha}^{2,1}$, will be called a Shigekawa-Hilbert-Sobolev space of order 1 on $\mathcal{D}_{1}^{*}(X, \mathcal{G})$.

Notice that if we should have taken $\alpha=0$, the corresponding Hilbert space would be $L_{F, 0}^{2,1}=L_{F}^{2}$.

\section{The regular representations $R_{\alpha}^{F}$ of the current group $\mathcal{D}(X, G)$}

\section{The regular representation $R_{0}^{F}$ on $L_{F}^{2}$.}

Let $F$ be a Riemannian flag of $X$. In I-2, proposition 2 we have seen that the action of the current group $\mathcal{D}(X, G)$ on $\mathcal{D}_{1}^{*}(X, \mathcal{G})$ given by the representation $V^{*}$ leaves invariant the energy Gaussian measure $\mu_{F}$. It follows that for all $g$ in $\mathcal{D}(X, G)$ the operators $R_{0}^{F}(g)$ defined for all $\Phi$ in $L_{F}^{2}$ by:

$$
\left(R_{0}^{F}(g) \Phi\right)(\chi)=\Phi\left(V^{*}\left(g^{-1}\right) \chi\right), \quad \chi \in \mathcal{D}_{1}^{*}(X, \mathcal{G}),
$$

are unitary operators on $L_{F}^{2}=L_{F, 0}^{2,1} ; R_{0}^{F}: g \mapsto R_{0}^{F}(g)$ is then a continuous unitary representation of $\mathcal{D}(X, G)$ on $L_{F, 0}^{2,1}$.

Definition 9. The unitary representation $R_{0}^{F}$ will be called the regular representation of $\mathcal{D}(X, G)$ on $L_{F}^{2}$. 
Proposition 12. For all $n$ in $\mathrm{N}$ the closed subspace $Z_{n}^{F}$ is invariant by $R_{0}^{F}$ and for all $g$ in $\mathcal{D}(X, G): R_{0}^{F}(g) \cdot p_{n}^{F}=p_{n}^{F} \cdot R_{0}^{F}(g)$.

Proof: Let $\omega$ be in $\mathcal{D}_{1}(X, \mathcal{G})$, and let $q_{w}$ be the polynomial Wiener functional $\chi \mapsto<\chi, \omega\rangle$; for all $g$ in $\mathcal{D}(X, G), R_{0}^{F}(g) q_{\omega}(\chi)=\left\langle V^{*}\left(g^{-1}\right) \chi, \omega\right\rangle$ $=\langle\chi, V(g) \omega\rangle=q_{V(g) \omega}(\chi)$. It follows that each Fourier-Hermite polynomial Wiener functional is transformed by $R_{0}^{F}(g)$ into a Fourier-Hermite polynomial Wiener functional with same degree. Therefore, from proposition 5 it follows that $Z_{n}^{F}$ is invariant by $R_{0}^{F}(g)$; this proves the assertion.

As a direct consequence of proposition 12 one gets:

Corollary 1. Let $\varphi$ be any mapping from $\mathrm{N}$ into $\mathrm{R}$ and let $T_{\varphi}$ be the operator: $T_{\varphi}=\Sigma_{n=0}^{\infty} \varphi(n) p_{n}^{F}$. For all $g$ in $\mathcal{D}(X, G)$ one has the commutation:

$$
R_{0}^{F}(g) \cdot T_{\varphi}=T_{\varphi} \cdot R_{0}^{F}(g)
$$

In particular:

Corollary 2. On P; all $M$-transforms and all generalized Cauchy operators $C^{F, \alpha}$ commute with the operators $R_{0}^{F}(g), \alpha \geq 0$.

2. The regular representation $R_{\alpha}^{F}$ on $L_{F, \alpha}^{2,1}, \alpha>0$.

Let $\alpha$ be in $\mathrm{R}^{+*}$, and for all $g$ in $\mathcal{D}(X, G)$ let $R^{F}(g)$ be the restriction to $P$ of the unitary operator (with respect to $\left.<,>_{L^{2}}\right) R_{0}^{F}(g)$; we shall denote by $R^{F}$ the mapping $g \mapsto R^{F}(g)$.

Proposition 13. $R^{F}$ extends into a continuous unitary representation of $\mathcal{D}(X, G)$ on the Shigekawa-Hilbert-Sobolev space $L_{F, \alpha}^{2,1}$ that we will denote $R_{\alpha}^{F}$ and call the regular representation of $\mathcal{D}(X, G)$ on $L_{F, \alpha}^{2,1}$.

Proof: Let us recall, firstly, that $P$ is contained in the domain of $L^{F, \alpha}$ and $C^{F, \alpha}$. We have to prove now that the operators $R^{F}(g), g \in \mathcal{D}(X, G)$, are unitary with respect to $\left\langle,>_{L_{\alpha}^{2,1}}\right.$.

Let $\Phi$ and $\psi$ be in $P$; for all $g$ in $\mathcal{D}(X, G)$ one has

$$
\begin{aligned}
& <R^{F}(g) \Phi, R^{F}(g) \psi>_{L_{\alpha}^{2,1}}=<R^{F}(g) \Phi, R^{F}(g) \psi>_{L^{2}}+ \\
& <C^{F, \alpha} \cdot R^{F}(g) \Phi, C^{F, \alpha} \cdot R^{F}(g) \psi>_{L^{2}}
\end{aligned}
$$

from the unitary of $R^{F}(g)$ with respect to $<,>_{L^{2}}$ it follows that

$$
<R^{F}(g) \Phi, R^{F}(g) \psi>_{L^{2}}=<\Phi, \psi>_{L^{2}} .
$$


On the other side, from corollary 2 of proposition 12 one has:

$$
\begin{aligned}
<C^{F, \alpha} \cdot R^{F}(g) \Phi, C^{F, \alpha} \cdot R^{F}(g) \psi>_{L^{2}}= & <R^{F}(g) \cdot C^{F, \alpha} \Phi, R^{F}(g) \cdot C^{F, \alpha} \psi>_{L^{2}} \\
& <C^{F, \alpha} \cdot \Phi, C^{F, \alpha} \cdot \psi>_{L^{2}}
\end{aligned}
$$

From the definition of $\langle,\rangle_{L_{\alpha}^{2,1}}$, and from $\left(^{*}\right)$ and $\left(^{* *}\right)$ it follows therefore that:

$$
<R^{F}(g) \Phi, R^{F}(g) \psi>_{L_{\alpha}^{2,1}}<\Phi, \psi>_{L_{\varepsilon}^{2,2}}
$$

Consequently one easily concludes that $R^{F}$ extends to a continuous unitary representation of $\mathcal{D}(X, G)$ on $L_{F, \alpha}^{2,1}$.

Remarks. On $P \cap Z_{1}^{F}$, i.e. on the space of polynomial Wiener functionals of degree $1, C^{F, \alpha}$ restricts to the identity so that the completion of $P \cup Z_{1}^{F}$ with respect to the scalar product $\left\langle,>_{L_{\alpha}^{2,1}}\right.$ is exactly $Z_{1}^{F}$, for all $\alpha$ in $\mathbf{R}^{+*}$; therefore $R_{0}^{F}$ and $R_{\alpha}^{F}$ are equal on $Z_{1}^{F}$. This is, of course, no more true for subspaces $P \cap Z_{n}^{F}$, with $n>1$.

2) To my knowledge, there is no equivalent phenomenon for regular representations of finite dimensional Lie groups.

\section{Elementary energy representations of the current group $\mathcal{D}(X, G)$}

1. On unitary action of $\mathcal{D}_{1}(X, \mathcal{G})$ on $L_{F}^{2}$.

a) Let $F$ be a Riemannian flag of $X$, and for all $\omega$ in $\mathcal{D}_{1}(X, \mathcal{G})$ let us consider the translation $T_{w}^{F}$ on $\mathcal{D}_{1}^{*}(X, \mathcal{G}): \chi \mapsto \chi+\omega$, which is such that for all $\theta$ in $\mathcal{D}_{1}(X, \mathcal{G}),\left\langle T_{\omega}^{F} \chi, \theta\right\rangle=\langle\chi, \theta\rangle+\langle\omega, \theta\rangle_{F}$. A well-known result about quasi-invariance of Gaussian measures by translations (see e.g. [9], [5]), allows to assert that $\mu_{F}$ is quasi-invariant by $T_{w}^{F}$, the corresponding Radon-Nikodym derivative being given by:

$$
\frac{d\left(T_{\omega}^{F} \circ \mu_{F}\right)(\chi)}{d \mu_{F}(\chi)}=\exp \left[-<\chi, \omega>-\frac{1}{2}|\omega|_{F}^{2}\right] .
$$

An immediate consequence of this is the following:

Proposition 14. For each $\omega$ in $\mathcal{D}_{1}(X, \mathcal{G})$ the mapping $U_{\omega}^{F}$ defined for all $\Phi$ in $L_{F}^{2}$ by:

$$
U_{\omega}^{F} \Phi(\chi)=\exp \left[-\frac{1}{2}\langle\chi, \omega\rangle-\frac{1}{4}|\omega|_{F}^{2}\right] \cdot \Phi(\chi+\omega),
$$

$\chi \in \mathcal{D}_{1}^{*}(X, \mathcal{G})$, is a unitary operator on $L_{F}^{2}$, and $U^{F}: \omega \mapsto U_{\omega}^{F}$ is a continuous unitary representation of the abelian nuclear Lie group $\mathcal{D}_{1}(X, \mathcal{G})$ on $L_{F}^{2}$. 
Notice that $U^{F}$ is highly reducible; but contrarily to $R_{0}^{F}$ this representation doesn't leave invariant the subspaces $Z_{n}^{F}, n \in \mathrm{N}$.

b) Let us recall that the Fourier-Wiener transform $\mathcal{J}^{F}$ is a unitary operator on $L_{F}^{2}$ (III-2); it follows that for all $\omega$ in $\mathcal{D}_{1}(X, \mathcal{G})$ the operator $W_{\omega}^{F}=$ $\mathcal{J}^{F} U_{\omega}^{F} \cdot\left(\mathcal{J}^{F}\right)^{-1}$ is also a unitary operator on $L_{F}^{2}$; therefore:

$$
W^{F}: \omega \mapsto W_{\omega}^{F}, \quad \omega \in \mathcal{D}_{1}(X, \mathcal{G}),
$$

is a unitary representation of $\mathcal{D}_{1}(X, \mathcal{G})$ on $L_{F}^{2}$ unitarily equivalent to $U^{F}$.

Proposition 15. The unitary representation $W^{F}$ is given for all $\omega$ in $\mathcal{D}_{1}(X, \mathcal{G})$ and all $\Phi$ in $L_{F}^{2}$, by:

$$
W_{\omega}^{F} \Phi(\chi)=\exp \left[-\frac{i}{2}<\chi, \omega>\mid \Phi(\chi), \quad \chi \in \mathcal{D}_{\perp}^{*}(X, \mathcal{G})\right.
$$

Proof: It follows from (32), (16), and an easy computation we leave to the reader.

\section{The energy representations $\pi^{F}$ of $\mathcal{D}(X, G)$.}

Such representations have been considered and studied in [14]; they constitute an enlargement of a class of non located unitary representations of order 1 of $\mathcal{D}(X, G)$ originally considered and studied by Ismagilov $([6])$ and by Gelf'and, Graev and Vershik ([23]). They are gotten by the following way.

Let $F$ be a Riemannian flag of $X$; the corresponding energy representation $\pi^{F}$ of $\mathcal{D}(X, G)$ on $L_{F}^{2}$ is such that for all $g$ in $\mathcal{D}(X, G)$, all $\Phi$ in $L_{F}^{2}$ :

$$
\begin{aligned}
& \pi^{F}(g) \Phi(\chi)=\exp \left[i<\chi, d g \cdot g^{-1}>\right] \Phi\left(V^{*}\left(g^{-1}\right) \chi\right), \\
& \chi \in \mathcal{D}_{1}^{*}(X, \mathcal{G}),
\end{aligned}
$$

where $g \mapsto d g \cdot g^{-1}$ denotes the so-called Maurer-Cartan cocycle ([23]).

Let $\underline{I}$ be the constant $L^{2}$-Wiener functional $\chi \mapsto I$ for all $\chi\left(\mu_{F}-\right.$ a.e. $)$. The following lemma summarize the properties of the energy representation $\pi^{F}$ associated to $F$, which are proved in [14]:

\section{Lemma 6 .}

(i) $\pi^{F}$ is a non located continuous unitary representation of order $l$ of $\mathcal{D}(X, G)$.

(ii) If $\operatorname{dim}(X) \geq 3$, 1 is a cyclic vector, $\pi^{F}$ is irreducible and two different Riemannian fiags $F, F^{\prime}$ give rise to inequivalent energy representations $\pi^{F}, \pi^{\prime}$.

(iii) For $\operatorname{dim}(X)=1, \pi^{F}$ is always reducible.

Let us add that, at the present time, the case $\operatorname{dim}(X)=2$ is not completely elucidated. 


\section{The elementary energy representations $\Gamma^{F}$.}

Let $F=\left(X_{k}, r_{k}\right)_{0 \leq k \leq p}$ be a Riemannian flag of $X$, and for all $g$ in $\mathcal{D}(X, G)$ let us define the operator $\Gamma^{F}(g)$ on $L_{F}^{2}$ by:

$$
\Gamma^{F}(g)=R_{0}^{F}(g) \cdot W_{d g \cdot g^{-1}}^{F},
$$

where $R_{0}^{F}$ is the regular representation of $\mathcal{D}(X, G)$ on $L_{F}^{2}$, and $W^{F}$ the unitary representation of $\mathcal{D}_{1}(X, \mathcal{G})$ given by (33) and proposition 15 . One gets, for all $\Phi$ in $L_{F}^{2}$, all $\chi$ in $\mathcal{D}_{1}^{*}(X, \mathcal{G}):$

$$
\Gamma^{F}(g) \Phi(\chi)=\exp \left[-\frac{i}{2}<V^{*}\left(g^{-1}\right) \chi, d g \cdot g^{-1}>\right] \cdot \Phi\left(V^{*}\left(g^{-1}\right) \chi\right) .
$$

Taking into account the fact that the Maurer-Cartan cocycle $g \mapsto d g \cdot g^{-1}$ is a continuous 1-cocycle of $\mathcal{D}(X, G)$ for $V$, and that $R_{0}^{F}$ and $W^{F}$ are continuous unitary representations of $\mathcal{D}(X, G)$ and $\mathcal{D}_{1}(X, \mathcal{G})$ respectively one gets:

Proposition 16. $\Gamma^{F}: g \mapsto \Gamma^{F}(g)$ is a continuous and non located unitary representation of order 1 of $\mathcal{D}(X, G)$ on $L_{F}^{2}$ :

Notice, from (35) and (37), that $\Gamma^{\vec{F}}$ and the energy representation $\pi^{F}$ have neighbouring forms; it is then natural to investigate the connexion between them.

To do this let us consider the unitary representation $\Gamma_{4}^{F}$ of $\mathcal{D}(X, G)$ in $\left(L_{F}^{2}\right)^{\otimes 4}$ defined for all $g$ in $\mathcal{D}(X, G)$ by:

$$
\Gamma_{4}^{F}(g)=\left(\Gamma^{F}(g)\right)^{\otimes 4}
$$

Proposition 17. $\Gamma_{4}^{F}$ is unitarily equivalent to the energy representation $\pi^{F}$,

Proof: It suffices to prove that $\Gamma_{4}^{F}$ and $\pi^{F}$ have a same spherical function.

The spherical function $s$ of $\pi^{F}$ with respect to the vector 1 is given by:

$$
\begin{aligned}
s(g)=<\pi^{F}(g) \underline{1}, \underline{1}>= & \int_{\mathcal{V}_{i}(X, \mathcal{G})} \exp \left(i<X, d g \cdot g^{-1}>\right) d \mu_{F}(X) \\
& =\exp \left(-\frac{1}{2}\left|d g \cdot g^{-1}\right|_{F}^{2}\right) .
\end{aligned}
$$

The spherical function $\sigma$ of $\Gamma_{4}^{F}$ with respect to $\underline{1}^{\otimes 4}$ is given by:

$$
\begin{aligned}
\sigma(g)= & \left.<\Gamma_{4}^{F}(g) \underline{1}^{\otimes 4}, \underline{1}^{\otimes 4}>=\left(<\Gamma^{F}(g) \underline{1}, \underline{1}\right\rangle\right)^{4} . \text { But : } \\
<\Gamma^{F}(g) \underline{1}, \underline{1}> & =\int_{\mathcal{D}_{i}^{*}(X, \mathcal{G})} \exp \left(-\frac{i}{2}<X, d g \cdot g^{-1}>\right) d \mu_{F}(X) \\
& =\int_{\mathcal{D}_{i}^{*}(X, \mathcal{G})} \exp \left(i<X,-\frac{1}{2} d g \cdot g^{-1}>\right) d \mu_{F}(X) \\
& =\exp \left(-\frac{1}{2}\left|\frac{1}{2} d g \cdot g^{-1}\right|_{F}^{2}\right)=\left[\exp \left(-\frac{1}{2}\left|d g \cdot g^{-1}\right|_{F}^{2}\right)\right]^{\frac{1}{4}} .
\end{aligned}
$$


We have then, for all $g$ in $\mathcal{D}(X, G)$ :

$$
\sigma(g)=\left(<\Gamma^{F}(g) \underline{1}, \underline{1}>\right)^{4}=\exp \left(-\frac{1}{2}\left|d g \cdot g^{-1}\right|_{F}^{2}\right)=s(g)
$$

which prove the assertion.

We notice that the equivalence of $\pi^{F}$ with $\left(\Gamma^{F}\right)^{\otimes 4}$ implies that when the energy representation $\pi^{F}$ is irreductible, then $\Gamma^{F}$ is necessarily irreducible.

Deffnition 10. The unitary representations $\Gamma^{F}$, where $F$ is any Riemannian flag of $X$, will be called the elementary energy representations of $\mathcal{D}(X, G)$.

\section{The rings of generalized energy representations of $\mathcal{D}(X, G)$}

\section{Motivations.}

At the present time the only non located unitary representations of $\mathcal{D}(X, G)$ we know and which are not trivially reducible are the elementary energy representations. The present section is devoted to the enlargement of such representations in order to get a ring of unitary, non located an order 1 (in the sense of [23], [12]) representations of the current group $\mathcal{D}(X, G)$ including the elementary energy representations as basic elements. A motivation for this enlargement comes from two points of view. On one hand it is necessary to have sufficiently non trivial and non located unitary representations of $\mathcal{D}(X, G)$ in hope to get a consistent non commutative harmonic analysis on spaces of generalized Wiener functionals; on the other hand we can also hope that such representation constitute a relevant tool in order to study $G$-valued stochastic variables and stochastic $G$-valued multiplicative measures ([1]).

Our construction is similar, in some sense, to the Weyl's construction of irreducible finite dimensional representations of the general linear group and its generalization given in $[7],[22]$ for finite functional dimension representations of the group of diffeomorphism of a non compact manifold.

\section{The generalized energy representation $\Gamma_{n, \rho}^{F}$.}

Let $F$ be a Riemannian flag of $X$ with associated energy Gaussian measure $\mu_{F}$ on $\mathcal{D}_{1}^{*}(X, \mathcal{G})$. For each positive integer $n$ we shall denote by $\mu_{F}^{\text {n }}$ the product measure $\mu_{F} \times \cdots \times \mu_{F}$ ( $n$ copies) on the product space $\mathcal{D}_{1}^{*}(X, \mathcal{G})^{n}$, and by $S_{n}$ the symmetric group of permutations of a set having $n$ elements; $\hat{S}_{n}$ will denote the set of (classes of) unitary representations of $S_{n}$. We have then a fibered set $\hat{S}=\bigcup_{n \leq 1} \hat{S}_{n}$ consisting of pairs $(n, \rho)$ where $n$ is a positive integer and $\rho$ a class of unitary equivalence of unitary representations of $S_{n}$ in some Hilbert space $E_{\rho}$.

To each element $(n, \rho)$ is $\hat{S}$ we associate the Hilbert space $L_{F}^{2}(n, \rho)$ of functionals $\Phi$ defined on $\mathcal{D}_{\mathrm{I}}^{*}(X, \mathcal{G})^{n}$ with values in the representation space $E_{\rho}$ of $\rho$ such that:

$$
\|\Phi\|_{F, n, \rho}^{2}=\int_{\mathcal{D}_{1}^{*}(X, G)^{n}}\left\|\Phi\left(\chi_{1}, \ldots, \chi_{n}\right)\right\|_{W_{\rho}}^{2} \cdot d \mu_{F}^{n}\left(\chi_{1} \ldots, \chi_{n}\right)<\infty .
$$


6. R. ISMAGILOV, On unitary representations of the group $C_{\mathfrak{0}}^{\infty}(X, G), G=$ $S U(2)$, Mat. U.S.S.R. Sb. 29 (1976), 105-117. (in Russian)

7. A.A. KIRIL LOV, Unitary representations of the group of diffeomorphisms and some of its subgroups, Preprint of the Inst. of Appl. Math. I.P.M. 82 (1974). (in Russian)

8. D. KNUTSON, " $\lambda$-rings and the representation theory of the symmetric group," Lect. Notes in Math. 308, Springer-Verlag, Berlin-Heidelberg-New York, 1973.

9. H.H. Kuo, "Gaussian measures in Banach spaces," Lect. Notes in Math. 463, Springer-Verlag, Berlin-Heidelberg-New York, 1975.

10. H.H. Kuo, Brownian functionals and applications, Acta Applic. Math. 1 (1983), 175-188.

11. S. KUSUOKA, D. STROOCK, Applications of the Malliavin calculus, part I, Proceedings of the Taniguchi International Symposium on Stochastic Analysis (1982), Kyoto, North-Holland.

12. J. MARION, $G$-distributions et $G$-Intégrales multiplicatives sur une variété, Annal. Pol. Math. 43 (1983), 79-92.

13. J. MARION, Dirichlet forms defined by Riemannian fiags and applications, Proc. 1 of the Ascona-Como International Meeting on stochastic processes in classical and quantum systems (1986).

14. J. MARION, D. TESTARD, Energy representations of gauge groups associated with Riemannian figs, Preprint No. 111, Research Center BielefeldBochum-Stochastics (1985), University of Bielefeld.

15. P.A. MEYER, "Quelques résultats analytiques sur le semi-groupe d'Ornstein-Uhlenbeck en dimension infinie," Lect. Notes in Control and Inf. Sci. 49, Springer-Verlag, Berlin-Heidelberg-New York, 1983.

16. G. SANSONE, Orthogonal functions, Interscience Publ. (1959), New York.

17. L. STREIT, T. HIDA, Generalized Brownian functionals and the Feynman integral, Stochastic processes and their applications, to appear.

18. I. SHIGEKAWA, Derivatives of Wiener functionals and absolute continuity of induced measures, J. Math. Kyoto Univ. 20, No. 2 (1980).

19. H. SugITA, Sobolev spaces of Wiener functionals and Malliavin's calcuius, J. Math. Kyoto Univ. 25, No. 1 (1985), 31-48.

20. H. SUGITA, On a characterisation of the Sobolev spaces over an abstract Wiener space, J. Math. Kyoto Univ. 25, No. 4 (1985), 717-725.

21. Y. UMEMURA, Measures on infinite dimensional vector spaces, $P u b$. Res. Inst. Math. Sci. Ser. A1 (1972), 1-47, Kyoto Univ. 
22. A.M. VERSHIK, I.M. GELF'AND, M.I. GRAEV, Representations of the group of diffeomorphisms, Russian Math. Surveys 30-6 (1975), 1-50.

23. A.M. VERSHIK, I.M. GELF'AND, M.I. GRAEV, Representations of the group of functions taking values in a compact Lie group, Comp. Math. 35 (1977), 299-336.

Dept. de Mathematiques, case 901 Faculté des Sciences de Luminy 70 Route Lëon Lachamp 13288 Marseille Cedex 9 FRANCIA

Rebut el 16 de maig de 1988 